\title{
An exploratory mixed methods study of urban and rural registered nurses' experience of clinical reasoning
}

\author{
Monique Sedgwick*1, Noelle Sedgwick ${ }^{1}$, Olu Awosoga ${ }^{1}$, Lance Grigg ${ }^{2}$, Sharon Dersch ${ }^{1}$ \\ ${ }^{1}$ Faculty of Health Sciences, University of Lethbridge, Lethbridge, AB, Canada \\ ${ }^{2}$ Faculty of Education, University of Lethbridge, Lethbridge, AB, Canada
}

Received: September 28, 2017

Accepted: November 28, 2017

Online Published: December 4, 2017

DOI: $10.5430 /$ jnep.v8n4p70

URL: https://doi.org/10.5430/jnep.v8n4p70

\begin{abstract}
Background and objective: Engaging in clinical reasoning frequently occurs in busy, high pressured, stressful settings with competing demands. Patient outcomes are affected in part by RNs' clinical reasoning ability. This study aims to explore the extent to which the clinical context influences clinical reasoning among urban and rural registered nurses.

Methods: In this exploratory study using a mixed method approach, 11 rural hospital RNs and 7 RNs practicing in urban medical or surgical units completed a survey and a semi-structured individual qualitative interview. Data were generated over a two month period in 2015. Descriptive statistics and Mann-Whitney U was used to test for differences among groups. Qualitative data analysis procedures were used to help identify two major themes.

Results: The perceived lack of time influenced the participants' ability to engage in clinical reasoning. The findings also suggest that rule following hampered the participants' ability to confidently share their clinical reasoning.

Conclusions: To deepen RNs clinical reasoning an examination of the clinical environment's structure and processes that support or impede engagement in clinical reasoning is required. Specific strategies that enhance clinical reasoning need to be unit specific and driven by RNs.
\end{abstract}

Key Words: Clinical reasoning, Registered nurses, Rural, Urban

\section{INSTRUCTION}

Clinical reasoning involves: professional knowledge (what nurses know) and action based on that knowledge (what they do with that knowledge in clinical situations). ${ }^{[1,2]}$ In her seminal work, Benner ${ }^{[3]}$ suggests that the ability to understand characteristics of current and potential future situations is important. As such, a plan of care must be based on conscious, abstract, and analytical contemplation of the problem and thus is closely linked to clinical reasoning ${ }^{[3]}$ Since Benner's ground breaking work further research suggests that nurses' reasoning skills are also contextually influenced. ${ }^{[4]}$ Indeed, it would appear that context specific knowledge as well as a broad experience base seem to be necessary for professional competence. ${ }^{[5]}$ Given that clinical reasoning provides the foundation for being able to provide appropriate care at the appropriate time, administrators and professional nurses ought to be concerned about how clinical reasoning might be enhanced. Since there is evidence that clinical reasoning is context sensitive, this study set out to explore how registered nurses (RNs) ability to engage in clinical reasoning

\footnotetext{
*Correspondence: Monique Sedgwick; Email: Monique.sedgwick@uleth.ca; Address: Faculty of Health Sciences, University of Lethbridge, Lethbridge, AB, T1K 3M4, Canada.
} 
was influenced by their clinical practice environment.

\subsection{Problem statement}

Engaging in clinical reasoning often times occurs in busy, high pressured, stressful clinical settings with competing demands. Such an environment may impact RNs' ability to engage in clinical reasoning that potentially can affect patient outcomes. ${ }^{[1,6]}$ A review of the nursing literature suggests that nursing practice environments have interrelated, multidimensional organizational factors that impact the practice environment. ${ }^{[7]}$ Two broad types of practice environments reported in the literature are the bureaucratic and professional models. At the point of care, the bureaucratic model is task centered, emphasizing hierarchical authority and control, and formal rule enforcement. The professional model emphasizes individual qualifications, accountability, empowerment and collegial team work. ${ }^{[8,9]}$ This type of model produces positive practice environments where nurses are respected and involved in decision-making. ${ }^{[10]}$

Rooted within the practice environment are unwritten rules regarding acceptable ways members are expected to perceive, think and feel. ${ }^{[11]}$ It seems that current nursing practice environments tend to reflect the more bureaucratic practice model where conformity and acceptance of 'this is way things have always been done' ${ }^{[7]}$ stifles nurses' professional responsibility to engage in reflective, evidence informed clinical reasoning. Consequently, transforming these environments needs to be a priority. ${ }^{[9]}$ However, exploring how the clinical environment influences RNs' ability to engage in clinical reasoning is necessary and relevant.

\subsection{Literature review}

CINHAL, ProQuest Nursing \& Allied Health Source, and MEDLINE databases were searched for articles between 1985 to the present. Initial search terms included: clinical reasoning, decision-making, practice setting, context, and work setting. Studies that investigate nurses' clinical reasoning fall into the following general categories: 1) ethical decision making; ${ }^{[12,13]}$ 2) clinical reasoning for specific nursing procedures $;^{[14,15]} 3$ ) decision-making and specific illnesses; ${ }^{[16,17]}$ 4) decision-making within specific practice settings; ${ }^{[18,19]}$ and 5) decision-making in nursing using simulation. ${ }^{[20,21]}$

Contextual factors that influence clinical decision making that have been studied include: education, ${ }^{[5,22,23]}$ experience, ${ }^{[3,24]}$ level of appointment, ${ }^{[18]}$ age, ${ }^{[25,26]}$ and occupational orientation. ${ }^{[27]}$ Thompson and Sutton ${ }^{[28]}$ found that factors (listed in order of priority) that affected clinical reasoning in critical care nurses included knowledge and experience, role modelling and least important, values. The order of priority for factors affecting clinical reasoning in Hughes

Published by Sciedu Press and Young's ${ }^{[29]}$ study were clinical experience, clinical setting, beliefs and preferences, short-term memory capacity and interpersonal conflict. In a more recent study, ${ }^{[4]}$ using the method 'participant objectivation', the researchers conducted 12 structured non-participant observations of admission assessments by four nurses. Although a small study, the researchers concluded that competence which is closely linked to clinical reasoning, is related to individual and contextual factors as opposed to length of experience. Depending on various environmental factors, patterns of practice seemed to be situationally rooted resulting in inexperienced nurses demonstrating deep clinical reasoning skills while experienced nurses demonstrated more superficial clinical reasoning skills in some situations. In a quantitative correlational survey study, ${ }^{[27]}$ the researchers found that nurses who worked on medical units engaged in decision-making more often and that education and experience were not related to clinical decision-making. However, in an integrative literature review of the factors that contribute to professional nurses and midwives making sound clinical decisions, tenHam, Ricks, vanRooyen and Jordan ${ }^{[30]}$ found that clinical knowledge and training was the most significant influential factor on clinical decision making followed by organizational factors and patient characteristics. Among environmental factors, time and material resources were factors that could influence clinical decision making aptitudes.

Based on this review, there appears to be some inconsistencies in the literature pertaining to how context influences clinical reasoning. In short, the identification of key elements of professional practice contexts that positively or negatively impact clinical reasoning in nursing is lacking in the current literature.

\subsection{Theoretical framework}

The terms 'critical thinking' and 'clinical reasoning', 'decision-making' and 'clinical judgment' are frequently used interchangeably in the literature. While there are similarities among these concepts, for example they all include elements of process and outcome, there are some important differences. To shed light on these differences, a brief comparison of critical thinking and clinical reasoning is offered.

Critical thinking is described as a cognitive process that includes rational analysis of information to facilitate clinical reasoning, judgment and decision making. ${ }^{[31]}$ Critical thinking suggests there in a goal or objective to thinking processes. ${ }^{[32]}$ Clinical reasoning on the other hand, emphasizes the processes prior to the end point. Clinical reasoning is made up of cognitive, meta-cognitive (reflective thinking), and discipline-specific knowledge (including contextual parameters of the patient and environment). The main purpose 
is to gather and analyze patient information, evaluate its significance, and weigh alternative actions. ${ }^{[32]}$ Clinical reasoning is dynamic in nature since it encourages the nurse to consider new information, interventions and alternative courses of action at multiple points during interactions with patient and healthcare team members.

Consistent with Simmons' description, ${ }^{[32]}$ in this study, clinical reasoning was understood as 'mindful doing'. ${ }^{[33]}$ As such, RNs use their cognitive and meta-cognitive skills to engage in clinical reasoning. Clinical reasoning was also understood to be responsive to significant social, psychological, cultural, and contextual influences. ${ }^{[34]}$ It includes the processes of collecting cues, processing information, coming to an understanding of the patient's situation, planning and implementing interventions, evaluating the outcomes of the interventions, and reflecting on and learning from the process. ${ }^{[35]}$ As highlighted previously, the purpose of this study was to explore how the practice context influenced clinical reasoning skills among urban acute care RNs and rural hospital RNs. Given the number of elements central to clinical reasoning, understanding the potential impact of the practice context is deeply relevant.

\section{METHOD}

\subsection{Design}

This study was an exploratory study using a concurrent mixed methods approach. RNs self-reported their clinical-decision making ability through the completion of a structured survey, and completed a 30 minute fully-simulated standardized clinical scenario followed by a qualitative semi-structured individual interview.

\subsection{Participants}

RNs working in urban acute medical or surgical care settings as well as RNs working in rural hospitals that offer acute care nursing services were recruited for this study. Recruitment for the study was limited to the healthcare zones in southern Alberta, Canada. To be included in the study, the participants had to be RNs who were working 0.5 full time equivalence (FTE) or greater, and have a minimum of one year post-graduation work experience in their practice setting. Participants self-identified as either being rural or urban. That being said, rural was generally considered to be communities of less than 10,000 people that required a minimum of three quarters of an hour drive to access amenities in cities with a population of $>100,000$ people.

The study sample consisted of 11 rural hospital RNs and 7 urban hospital RNs. Of the seven participants who identified their work setting as being either medical or surgical units in urban hospitals, $85 \%$ of them worked on general medical units and $15 \%$ worked on general surgical units. All of the participants who worked in rural hospitals described their practice setting as offering limited specialized medical and ancillary services (for example physio therapy and respiratory therapy) where nursing care was provided to patients of all ages and who were experiencing a wide variety of health concerns. Urban participants described their work environment as being fast paced. They provided care to adults experiencing a variety of illnesses with varying degrees of acuity. Extensive ancillary services were available to them on days and evenings with fewer services readily available on night shift.

Seventeen participants were female and one participant was male. They ranged in age between 20 and older than 40 years of age with $45 \%$ of the sample being between 26 and 35 years of age. One participant held a graduate degree, five had completed a diploma in nursing, and twelve participants held a university undergraduate degree. Over half of the participants $(67 \%)$ had completed their formal nursing education program within the last five years. Consequently, the majority of the participants had five years or less of work experience.

\subsection{Research setting}

According to Nosich, ${ }^{[33]}$ reasoning does not occur in a void. Rather, context provides the background to reasoning. Moreover, while the context shapes which alternative courses of action are possible, alternative courses of action also shape the context. Identifying each the purpose for engaging in reasoning, the main question pertaining to the event, assumptions being made regarding the context, patient, and colleagues, implications and consequences associated with actions, professional concepts that guide RN practice, conclusions that are a result of the action taken or not taken, and point of view is essential to engaging in sound reasoning. This being said, personal experience with engaging in deep critical reasoning, as well as the context in which this reasoning occurs, influences the experience of thinking things through.

To provide participants with an environment in which they could engage in clinical reasoning, we created a fully simulated environment as well as a standard scenario that the participants worked through. The advantages to creating a simulated but realistic environment in which the participants could engage in clinical reasoning included: 1) providing the researchers with comparable data sets thus enhancing reliability and validity of the findings; 2) providing the researchers with a degree of control over specific elements that were of particular interest to the research study and; 3) avoiding clinical problems that may raise ethical dilemmas and potentially 
place patients at risk.

With the assistance of expert rural hospital nurses as well as nurses with expertise in medical/surgical content, a scenario was developed. The scenario required the use of both high and low fidelity mannequins. The mannequins were programed using typical medical and/or surgical patient health conditions. For example, one mannequin was programed to represent a 76-year-old female with dementia and Type II Diabetes; another mannequin represented a patient with chest pain; and another mannequin exhibited the signs and symptoms of a patient with pneumonia. Further, each participant interacted with three actors (RNs) at various time during the scenario. All the actors followed a script associated with their role which included a night nurse, a physician, and a Licensed Practical Nurse. The simulation coordinator controlled the computer programs for the high fidelity mannequins.

\subsection{Ethical considerations}

Ethics approval was received from the university Human Subject Research Committee (File \#2014-072). An invitation to participate in the study was forwarded to the nursing staff by unit managers and through the researchers' professional networks. Potential participants were provided with a letter of invitation outlining the research procedures and researcher contact information. If the potential participant had further questions, these were answered by the Principle Investigator prior to booking a time for data collection. Participation was voluntary. Although all of the participants were made aware of their right to withdraw from the study at any time, all of the participants completed the study. Written informed consent was obtained after all of the research activities were explained, and prior to the start of data collection. Consent was further verified following completion of the scenario and prior to the interview. All quantitative data were anonymized. Transcripts were assigned pseudonyms. Following the guidelines for ethical management of research documents, all project documents are kept in a locked cabinet and password protected computers. Data were generated over a two month period in the summer of 2015.

\subsection{Data collection}

\subsubsection{Study procedures}

Prior to working through the scenario, the participants completed the Clinical Decision Making in Nursing Scale (CNMNS) ${ }^{[36]}$ and Clinical Effectiveness and Evidence Based Practice Questionnaire (EBPQ) ${ }^{[37]}$ Once the participant had completed the questionnaire, an orientation to the simulated nursing unit was provided by the simulation coordinator. The participant then listened to a 'taped report' of the previous shift (typical handoff procedure on nursing units). After they had listened to 'report' and had completed their workflow sheet (each participant made a workflow sheet they normally used at their workplace), they were escorted to the simulation lab where they worked through the standardized 30 minute clinical scenario.

To reduce the time commitment for participating in the study and reduce the number of participants dropping out of the study, the participants were interviewed immediately after they had completed the scenario in an adjacent room to the laboratory.

\subsubsection{Survey questionnaires and interview}

The $\mathrm{CDMNS}^{[36]}$ is a 40 -item questionnaire with a 5-point Likert-type (from never to always) response scale with higher score values interpreted as having a more positive perception of decision-making. It examines the respondents' selfperception of their decision-making abilities by assessing four categories of decision-making: search for alternative courses of action; surveying of objectives and professional values; evaluation and re-evaluation of courses of action and; search for information and unbiased assimilation of new information.

The $\mathrm{EBPQ}^{[37]}$ is a 25-item 7-point Likert-type response scale. This instrument is intended to explore nurses' day to day use of evidence-based practice. Cronbach's alpha for the former was 0.83 and 0.87 for the latter. Both instruments have been extensively used in other research studies and support the focus of this study which is to explore how the practice context might influence clinical reasoning skills among urban acute care RNs and rural hospital RNs.

The questions asked during the semi-structured interview were based on elements of reasoning ${ }^{[33]}$ and are found in Table 1. All interviews were digitally recorded and transcribed verbatim for analysis. The interviews lasted between 60 and 90 minutes.

\subsection{Data analysis}

Quantitative and qualitative data were collected concurrently in this exploratory study. Each data set was analyzed independently of the other data set with analysis occurring concurrently. Once analysis of each data set was completed, the authors assessed whether the results of the two data sets were congruent or divergent. ${ }^{[38]}$ The merged results are presented in the discussion section.

\subsubsection{Quantitative data analysis}

Data were analyzed using the Statistical Package for the Social Sciences (SPSS) v.22. Descriptive statistics were obtained for all survey items. The Mann-Whitney U test was used to test for differences among rural and medical/surgical 
urban hospital RNs. The significance level $(\alpha)$ was set at 0.05 . Cronbach alpha was used to complete the reliability analysis of the questionnaire (CDMNS: .741; EBPQ: .806). A G*Power 3.1.9.2 software was used to conduct a sensitivity analysis to estimate the required sample size and power of the test. For a linear bivariate regression for two groups (Urban/Rural) difference between intercepts, setting alpha $=0.05$ and power $=0.80$ a minimum sample size of $16(\mathrm{n} 1$
$=10, \mathrm{n} 2=6$ ) was required. The actual power obtained was 0.8352. For a correlation (Point biserial model), setting alpha $=0.05$, power $=0.80$, a minimum sample size of 18 would require a moderate effect size of 0.52 . For a linear bivariate regression for two groups (Urban/Rural) difference between slopes, setting alpha $=0.05$ and power $=0.80$ a minimum sample size of $18(\mathrm{n} 1=11, \mathrm{n} 2=7)$ was needed. The actual power obtained was 0.8038 .

Table 1. Interview questions

\begin{tabular}{ll}
\hline Element of Reasoning & Questions \\
\hline $\begin{array}{l}\text { Questions identifying the } \\
\text { question-at-issue }\end{array}$ & What were some questions you believed you had to be answered the scenario? \\
Questions about the purpose & What was the most important question you believed you had to answer in the scenario? \\
& What were some tasks you believed you had to do in the scenario? \\
& What was the main task you believed you had to do in the scenario? \\
Questions about assumptions made & Were you aware of any assumptions you were making regarding nursing while you \\
& were working through the scenario? \\
& Did you question any assumptions you made during the scenario? \\
Questions about the context & What was happening in the scenario? Why was this important to you? \\
& How aware of the context were you? \\
Questions about implications and & How important is the context for you for completing nursing tasks? \\
consequences & What were the possible implications or consequences of your decisions? \\
Questions about professional concepts & How did these implications or consequences impact your decision about what to do? \\
& What were some nursing concepts that you used in the scenario? \\
Questions about conclusions & What major conclusion did you come to in order to provide safe, efficient care? \\
& What information did you use to support these reasons? \\
Questions about information & Were your decisions the best decisions possible given the situation? Why? Why not? \\
Questions about alternatives & What information was important or meaningful to you in this scenario? \\
\hline
\end{tabular}

\subsubsection{Qualitative data analysis}

A template that included participant verbatim responses according to the elements of reasoning and standards of reasoning ${ }^{[33]}$ and the analysis was created. In order to identify differences if any, among rural and urban RNs, each interview was analyzed line by line by the authors of this article. Consensus among the researchers was used to ensure consistency of the analyses (i.e. dependability) and credibility. ${ }^{[39]}$ Credibility was established through the use of a simulated scenario and interview questions that had been used in a previous study. Use of a mixed methods approach also helped to establish credibility since the data generated in this study represents both qualitative and quantitative findings and so provide a more complete and contextualized portrait of how RNs' ability to engage in clinical reasoning was influenced by their practice environment. ${ }^{[40]}$ Transferability is established through thick, rich descriptions that novice nurses who work in other practice settings recognize as their own experience. $^{[39]}$

\section{FindingS}

Qualitative data analyses for this sample, produced two major themes: 1) the predominance of rule-following and;2) a consistent perceived lack of time for task completion. These are supported by the quantitative data analysis that is provided at the end of each theme.

\subsection{The predominance of rule-following}

Both rural and urban participants described rule-following as the norm in their workplace environment. There were specific written and "unwritten rules", and a number of assumptions they had to follow. Unfortunately, some of these rules seemed to discourage the participants from seeking help:

I always think that they're busy doing something and I don't want to bother them (Clare, rural, 2 years of experience). 
For Alissa, asking for assistance was perceived as appropriate only when she was overwhelmed:

I don't like to put a bunch on the other people because I know that they have their stuff to do. If you're drowning in your pod you can always ask for help or if they're just sitting at the desk talking, you can always ask them to help out but... (Alissa, urban, 3 years of experience).

Along with following the rule of asking for assistance only under specific conditions, another rule the participants were expected to follow was ensuring that all relevant information had been gathered prior to approaching the team for consultation regarding the best course of action. Operating under this assumption is reflective of a more bureaucratic environment rather than a collegial one wherein evidence is identified and examined as a team:

It depends on the situation, and it depends on the support that I get. Like if I have a gut instinct but I don't have support from my colleagues then I have to go back to the evidence (Denise, urban, 3 years of experience).

Consequently, offering creative solutions was deemed appropriate only in certain, well-defined situations:

It's the rigidity of how a shift goes. There's no fluctuation between anything unless something kind of goes wrong. When chaos arises then your alternatives kick in but it is super rigid (Betty, urban, 4 years of experience).

If there's something emergent or really critical, everything else gets put on hold and we deal with the critical situation. If the unit clerk is gone I'll have somebody else fill out my transfer papers and somebody else will be doing the ECG and I'll be phoning the doctor or whatever. So like if it is a critical situation or an emergent situation we do divide up like that. But it has to be a pretty serious situation before we tend to do that, not just like I have heavy work load. You cope as long as you can with what you've got (Holly, rural, 4 years of experience).

For David, following rules was so thoroughly entrenched that breaking or veering away from them had significant consequences. Afraid of making mistakes, he grew suspicious of his own ability to engage in questioning and effective clinical decision-making:

Published by Sciedu Press
I'm also thinking I don't want to drop any balls, I don't want to be the weak link ... that stresses me out (David, rural, 5 years of experience).

The quantitative data analysis seems to support the notion that rural and urban workplace environments for these participants is rule-bound. For example, a negative correlation ( $r=$ $-.687, p=.002$ ) between the question 'Looking for new information in making a decision is more trouble than it's worth' (CDMNS Q\#4) and the question 'I use books or professional literature to look things up I don't understand' (CDMNS Q\#5), seems to suggest that maintaining the status quo and following rules is important. Similarly, there was a negative correlation $(r=-.618, p=.007)$ between the question 'Looking for new information in making a decision is more trouble than it's worth' (CDMNS Q\#4) and the question 'My clinical practice has changed because of evidence I have' (EBPQ Q\#10). It would appear that both these rural and urban participants did not engage in activities that would change their practice or how things were done on their unit of employment: they simply followed the rules. In short, the rules created to enhance the quality of nursing care became the focus rather than the nursing care itself. Hence, suggestions as to how to best improve practice by rule modification were rarely if ever shared.

\subsection{A consistent perceived lack of time for task comple- tion}

A perceived lack of time featured heavily in the participants' discussions about their ability to engage in clinical reasoning. For example, some participants suggested that the level of busyness on the nursing unit, as well the patient's level of acuity determined which course of action they would choose in the provision of nursing care. Because of a perceived lack of time, previous courses of action believed to be effective were used rather than considering whether an alternative course of action might be better. Time, rather than better courses of action, became the determining factor:

Sometimes you don't have time to do an alternative, like your patient's sick, this is what you need to do right now. Once you know how to do something, what do you have time for kind of thing that determines what you're going to do (Linda, rural, 2 years of experience).

Depending on time, sometimes you don't have time to start with the easiest most simple interventions. You go straight to the more aggressive interventions (Holly, rural, 4 years of experience). 
For other participants, the amount of time an intervention took determined perceptions of time available for task completion. For example, Marie ranked interventions, listing those her colleague might do so that she could complete other tasks needing her attention:

I knew I wanted an ECG and I thought if I can go get this nitro and give it to the patient, then the LPN would have time to do the ECG and I can be making the phone call. I thought it would free up a little bit more of my time to do what else I needed to do (Marie, rural, 15 years of experience).

Other participants described instances where they felt the organizational culture prevented them from taking time to consider alternative courses of action. The system got in their way. For example Holly describes the organizational culture in her place of employment as one wherein RNs are expected to work independently to the point where collaboration with team members was frowned upon. Nurses were expected to successfully complete tasks on their own. While respecting nursing autonomy, this can also nurture isolationism. In turn, this can impact a nurse's willingness to think collaboratively and explore alternative, better ways to care for patients:

That's a resource [referring to colleagues] that's untapped. I don't use help that might be available because I'm not used to doing that. Like you've got your five patients and I don't know what's going on with your five patients and you don't know what's going on with mine (Holly, rural, 4 years of experience).

To cope with the pressure of completing tasks in a timely fashion, some participants engage in 'multitasking' that might put patient care and outcomes at risk:

I just try to multitask properly. Like making sure that I give medications quickly and process stat orders (Alissa, urban, 3 years of experience).

For some participants, this perceived lack of time for task completion was stressful. Such stress made them doubt their ability to engage in sound clinical decision making and clinical reasoning. This creeping self-doubt made them regularly, second-guess themselves:

When people get very sick very quickly, is there a way I could have dealt with that situation better? Was there something that I missed that could have told me that he was about to decline that swiftly? What piece of information did I miss that maybe could have prevented it getting to that point? Did I respond quickly enough? What were some preventative things I could have done? (Gabby, urban, 5 years of experience).

So while all of the participants, regardless in which practice environment they worked in, demonstrated a willingness to improve their nursing practice which is reflective of deep clinical reasoning, some of the participants remained frustrated and unsure of their reasoning ability because of a perceived lack of time:

Being stretched in too many different directions and worrying and being crazy. I don't want to miss anything and I might not be able to be in all of the rooms at the right time (Martha, rural, $>20$ years of experience).

Statistically significant correlations between items pertaining to time and activities supporting deep clinical decision making were present in our quantitative analysis (see Table 2). Although caution is required in interpreting these findings due to the small sample size, the findings seem to support the notion that time is a factor in the participants' perceived ability to engage in critical reasoning. More precisely, the lack of time negatively impacts the ability to seek new information and consider alternatives in the provision of care.

Table 3 presents the correlations between the CDMNS four sub-categories (search for alternative courses of action; surveying of objectives and professional values; evaluation and re-evaluation of courses of action and; search for information and unbiased assimilation of new information) and the total EBPQ. As can be seen, there were no statistically significant findings. That being said, it is possible that the negative correlations capture the lack of time participants experienced during the simulation. That is, they may not have had enough time to evaluate their course of action and adjust their care accordingly. They might also have felt pressured to complete the tasks during the simulation they felt needed to be completed and did not have time to search for and incorporate new information in their plan of care.

\section{Discussion}

Accurate and sound clinical reasoning in health-care delivery relies heavily on what nurses know about their patients, their ability to engage patients in discussion about their needs, and the nurses' ability to engage with others. ${ }^{[41,42]}$ For the participants in this study, although they paid attention to the cues gathered from their patients, following rules and the perceived lack of time negatively impacted their willingness to 
(1) seek out new, relevant information; (2) engage in creative problem solving and; (3) discuss alternative courses of action with colleagues. Although the perceived lack of time for task completion might be related to the how the simulated scenario was structured, the perception of lack of time seemed to inhibit the participants' ability to satisfactorily complete day-to-day tasks. Unlike other studies where participants spent a considerable amount of time communicating their decisions to others, ${ }^{[18,42]}$ many of the participants in this study felt unable to collaborate with others despite being aware of the benefits of such collaboration. This may have lead some of the participants to question their competency in being able to work in acute, high-stressed environments with competing priorities and demands.

Table 2. Statistically significant correlation between CDMNS \& EBPQ questionnaire items (time)

\begin{tabular}{ll}
\hline & $\begin{array}{l}\text { If the clinical decision is vital and there is enough time, I } \\
\text { conduct a thorough search for alternatives (CDMNS Q\#1) }\end{array}$ \\
\hline $\begin{array}{l}\text { I go out of my way to get as much information as possible to make } \\
\text { decisions (CDMNS Q\#8) }\end{array}$ & $r=.471, p=.032$ \\
$\begin{array}{l}\text { The risks and benefits are the farthest thing from my mind when I } \\
\text { have to make a decision (CDMNS Q\#38) }\end{array}$ & $r=-.511, p=.021$ \\
$\begin{array}{l}\text { Critically appraised against a set criteria any literature you have } \\
\text { discovered (EBPQ Q\#3) }\end{array}$ & $r=.522, p=.03$ \\
$\begin{array}{l}\text { Integrated the evidence you found with your expertise } \\
\text { Evidence based practice is fundamental to professional practice } \\
\text { (EBPQ Q\#9) }\end{array}$ & $r=.484, p=.03$ \\
\hline
\end{tabular}

Note. $r$ is the correlation coefficient; $p$ is the probability value from the statistical test.

Table 3. Correlation between self-perception of decision making and total evidence-based practice

\begin{tabular}{ll}
\hline & $\boldsymbol{r} \boldsymbol{p}$ value \\
\hline Search for alternative courses of action & $r=.535, p=.033$ \\
Surveying of objectives \& professional values & $r=.399, p=.126$ \\
Evaluation \& re-evaluation of courses of action & $r=-.214, p=.427$ \\
Search for information and assimilation of new information & $r=-.106, p=.696$ \\
\hline
\end{tabular}

Note. $r$ is the correlation coefficient; $p$ is the probability value from the statistical test.

So while standardized nursing practice and habituated rulefollowing are important in certain nursing care contexts to ensure safe patient outcomes are consistently met, rules cannot be rigidly applied to complex nursing practice situations. ${ }^{[43]}$ Indeed, an overemphasis on rules and disengaged reasoning sanctions a context-insensitivity. The neglect of context wherein decisions are made further impedes the ongoing development of clinical reasoning competencies, and discourages nurses from developing attitudes of intellectual curiosity and perseverance.

\subsection{Implications}

Nurse work environments are complex organisms comprising of three different dimensions: culture, leadership and evaluation. ${ }^{[44]}$ Equally important sub-elements in each of these environments include: systems of decision-making, staff relationships, organizational systems, power differentials, and the potential of the organization to be innovative. ${ }^{[4]}$ Like any organism, nursing work environments are deeply interconnected, and change in one area can deeply impact all areas. With this caution as the backdrop for the discussion of the

Published by Sciedu Press application of the study findings, the following suggestions are offered as a way to enhance RNs attempts and ability to engage in deep contextually sensitive clinical reasoning.

Although Hauck, Winsett and Kuric's ${ }^{[46]}$ work focused on transformational leadership to achieve organizational change pertaining to evidence-based practice (EBP), lessons learned in their study are applicable in addressing the issues the participants in this study reported. For example, they suggest that for change to be successfully implemented, a broad vision of the structure and processes need to be in place. One such structure applicable to this current study is to have a facilitator whose role is to support nurses and reduce the barriers to implementing change. ${ }^{[47,48]}$ In this case, facilitators might look at scheduling practices, and ensure there is a balance between more and less experienced nurses working the same shift so that less experienced nurses can have the opportunity to discuss their reasoning with more experienced colleagues.

Another possibility is professional mentoring. Nurse leaders could become role models, mentoring other nurses in how 
to engage in clinical reasoning. This may be especially important for less experienced nurses like those in this study. Development of clinical reasoning competencies would help nurses, nurse leaders, and other healthcare professionals understand the nurses' role and responsibilities. One way of developing this type of competency is to deconstruct everyday events and reconstruct them while using elements of reasoning. ${ }^{[33]}$

Other suggestions pertaining to structure include managers encouraging nurses to spend the time they need with patients and to encourage nurses to offer suggestions on how to improve work flow thereby, being able to spend time getting to know their patients. ${ }^{[49]}$ More broadly, managers should be encouraged to account for variables in their environment they can change rather than focussing solely on organizational goals like patient satisfaction or revenue. ${ }^{[4]}$ Focus on relationship building among nurses and patients can also be of benefit to health care administrators and hospital managers.

An exploration of how nurses are involved in decisionmaking regarding how care is delivered, and tracking of patient outcomes may address the concern about lack of time. ${ }^{[50]}$ Further, examining whether nurses are able to work with superiors, peers, subordinates and other healthcare team members in a trusting environment based on shared values might help identify strategies that address the perceived need to follow rules.

\subsection{Limitations}

The subjectivity of self-reporting should be recognized although both questionnaires used in this study have been widely used, and their reliability and credibility have been established. Although the sample size is small and a large proportion of participants have five or less years of work experience limiting the generalizability of the findings, the findings identify contextual factors that might influence clinical reasoning particularly for RNs relatively new to nursing practice. Lastly, although recreating a fully simulated environment is time consuming and costly, using it as the 'field' avoids any concerns with conducting this type of inquiry in the field where 'real-life' patient outcomes hang in the balance.

\section{Conclusion}

Rule following and perceived lack of time were two themes that arose in this study. Indeed, the findings of this study suggest that participants' overemphasis on rules and disengaged reasoning produced for them a degree of context-insensitivity. To support the development of context sensitivity clinical reasoning ability, nurses need to be involved in organizational decision making. Consequently, they should be invited to collaborate in designing and implementing solutions to the issues on their unit ${ }^{[51]}$ resulting in deeper clinical reasoning ability and better patient outcomes.

\section{Conflicts of Interest Disclosure}

The authors declare that there is no conflict of interest.

\section{REFERENCES}

[1] Fonteyn ME, Ritter BJ. Clinical reasoning in nursing. In J. Higgs \& M. Jones (Eds.), Clinical reasoning in the health professions 2nd ed. (pp. 107-116). Woburn, MA: Reed Educational and Professional Publishing; 2010.

[2] Greenwood J, King M. Some surprising similarities in the clinical reasoning of 'expert' and 'novice' orthopaedic nurses: Report of a study using verbal protocols and protocol analyses. Journal of Advanced Nursing. 1995; 22: 907-913. PMid:8568065 https : //doi.org/10.1111/j.1365-2648.1995.tb02642.x

[3] Benner P. From Novice to Expert: Excellence and Power in Clinical Nursing Practice. Menlo Park, CA: Addison-Wesley; 1984.

[4] Rischel V, Larsen K, Jackson K. Embodied dispositions or experience? Identifying new patterns of professional competence. Journal of Advanced Nursing. 2008; 61: 512-521. PMid:18261060 https://doi.org/10.1111/j.1365-2648.2007.04543.x

[5] Andersson N, Klang B, Petersson G. Differences in clinical reasoning among nurses working in highly specialised paediatric care. Journal of Clinical Nursing. 2012; 21: 870-879. PMid:22239165 https://doi.org/10.1111/j.1365-2702.2011.03935.x
[6] Parsonage I. Making clinical decisions in emergency situations. Emergency Nurse. 2010; 18(4): 18-21. PMid:20662404 https : //doi.org/10.7748/en2010.07.18.4.18.c7902

[7] Martha S. Climate, culture, context, or work environment? Organizational factors that influence nursing practice. The Journal of Nursing Administration. 2000; 30(2): 53-58. https ://doi.org/10.1097/ 00005110-200002000-00002

[8] Lake ET. Development of the practice environment scale of the nursing work index. Research in Nursing \& Health. 2002; 25: 176-188. PMid:12015780 https ://doi.org/10.1002/nur.10032

[9] Lake ET, Friese CR. Variations in nursing practice environments. Relation to staffing and hospital characteristics. Nursing Research. 2006; 55(1): 1-9. https://doi.org/10.1097/00006199-200601000 $-00001$

[10] Canadian Nurses Association \& Canadian Federation of Nurses Unions (2015). Practice environments: Maximizing outcomes for clients, nurses and organizations. Available from: https://cna-aiic.ca/ /media/cna/page-content/pdf-e $\mathrm{n} /$ practice-environments-maximizing-outcomes-for-c lients-nurses-and-organizations_joint-position-sta tement.pdf?la=en 
[11] Leovaridis C, Cismaru DM. Characteristics of organizational culture and climate in knowledge-intensive organisations. Romanian Journal of Communications \& Public Relations. 2014; 16(2): 35-56. https ://doi.org/10.21018/rjcpr.2014.2.181

[12] Falco-Pegueroles A, Lluch-Canut T, Roldan-Merino J, et al. Ethical conflict in critical care nursing: Correlation between exposure and types. Nursing Ethics. 2015; 22(5): 594-607. PMid:25335920 https://doi.org/10.1177/0969733014549883

[13] Suzuki C, Ota K, Matsuda M. Information-sharing ethical dilemmas and decision-making for public health nurses in Japan. Nursing Ethics. 2015; 22(5): 533-547. PMid:25316458 https://doi.org/ 10.1177/0969733014549879

[14] Owen EB, Woods CR, O'Flynn JA, et al. A bedside decision tree for use of saline with endotracheal tube suctioning in children. Critical Care Nurse. 2016; 36(1): e1-10. PMid:26830187 https://doi.org/10.4037/ccn2016358

[15] Tingsvik C, Johansson K, Martensson J. Weaning from mechanical ventilation: Factors that influence intensive care nurses' decision-making. Nursing in Critical Care. 2015; 20(1): 16-24. PMid:25269708 https://doi.org/10.1111/nicc.12116

[16] Koppitz A, Bosshard G, Kipfer S, et al. Decision-making in caring for people with dementia at the end of life in nursing homes. International Journal of Palliative Nursing. 2016; 22(2): 68-75. http://doi.org/10.12968/ijpn.2016.22.2.68

[17] Palmer D. Shared decision making for patients living with inflammatory arthritis. British Journal of Nursing. 2016; 25(1): 3135. PMid:26768042 https://doi.org/10.12968/bjon. 2016 .25 .1 .31

[18] Bucknall TK. Critical care nurses' decision-making activities in the natural clinical setting. Journal of Clinical Nursing. 2000; 9: 25-36. PMid:11022489 https://doi .org/10.1046/j.1365-2702.20 00.00333. $\mathrm{x}$

[19] Taylor J, Sims J, Haines TP. The emergent relevance of care staff decision-making and situation awareness to mobility care in nursing homes: An ethnographic study. Journal of Advanced Nursing. 2014; 70(12): 2767-2778. PMid:24735067 https://doi.org/10.111 $1 / \mathrm{jan} .12425$

[20] Fomeris SG, Neal DO, Tiffany J, et al. Enhancing clinical reasoning through simulation debriefing: A multisite study. Nursing Education Perspectives. 2015; 38(5): 304-310. https ://doi.org/10.5480/ 15-1672

[21] Shelestak D, Meyers TW, Jarzembak J, et al. A process to assess clinical decision-making during human patient simulation: A pilot study. Nursing Education Perspectives. 2015; 36(3): 185-187. https://doi.org/10.5480/13-1107.1

[22] Sarsfield E. Difference between novices' and experts' solving illstructured problems. Public Health Nursing. 2014; 31(5): 444-453. PMid:24372564 https://doi .org/10.1111/phn.12100

[23] Turkel MC. Describing self-reported assessments of critical thinking among practicing medical-surgical registered nurses. MEDSURG Nursing. 2016; 25(4): 244-250.

[24] Stinson KJ. Benner's framework and clinical decision-making in the critical care environment. Nursing Science Quarterly. 2017; 30(1): 52-57. PMid:28899295 https://doi.org/10.1177/08943184 16680536

[25] Lew-Snider DM. Moral judgment, level of satisfaction with values conflict resolution, perceived intuitiveness and clinical decision making in nursing (Doctoral dissertation). Retrieved from ProQuest Dissertations \& Theses A \& I. 2003.

[26] Schutzenhofer KK, Musser DB. Nurse characteristics and professional autonomy. Image: Journal of Nursing Scholarship. 1996;
26: 201-205. https://doi.org/10.1111/j.1547-5069.1994 .tb00314.x

[27] Hoffman K, Donoghue J, Duffield C. Decision-making in clinical nursing: Investigating contributing factors. Journal of Advanced Nursing. 2004; 45(1): 53-62. PMid:14675301 https://doi .org/ $10.1046 / j .1365-2648.2003 .02860 . x$

[28] Thompson DR, Sutton TW. Nursing decision-making on a coronary care unit. International Journal of Nursing Studies. 1985; 22: 259266. https://doi.org/10.1016/0020-7489(85) 90008-2

[29] Hughes KK, Young W. The relationship between task complexity and decision-making consistency. Research in Nursing and Health. 1990; 13: 189-197. PMid:2188304 https://doi.org/10.1002/ nur. 4770130308

[30] TenHam W, Ricks E, vanRooyen D, et al. An integrative literature review of the factors that contribute to professional nurses and midwives making sound clinical decisions. International Journal of Nursing Knowledge. 2017; 28(1): 19-29. PMid:25982301 https://doi.org/10.1111/2047-3095.12096

[31] Alfaro-LeFevre R. Critical thinking, clinical reasoning, and clinical judgment: A practical approach 5th ed. St. Louis, MO: Saunders Elsevier; 2013.

[32] Simmons B. Clinical reasoning: Concept analysis. Journal of Advanced Nursing. 2010; 66(5): 1151-1158. https ://doi.org/10.1 $111 / j \cdot 1365-2648.2010 .05262 \cdot \mathrm{x}$

[33] Nosich GM. Learning to Think Things Through: A Guide to Critical Thinking Across the Curriculum 4th Ed. Pearson, New York, NY. 2012.

[34] Rashotte J, Carenvale F. Medical and nursing clinical decision making: A comparative epistemological analysis. Nursing Philosophy. 2004; 5: 160-174. PMid:15189556 https ://doi.org/10.1111/ j. 1466-769x. 2004.00175.x

[35] Levett-Jones T, Hoffman K, Dempsey J, et al. The 'five rights' of clinical reasoning: An educational model to enhance nursing students' ability to identify and manage clinically 'at risk' patients. Nurse Education Today. 2010; 30: 515-520. PMid:19948370 https://doi.org/10.1016/j.nedt.2009.10.020

[36] Jenkins HM. Clinical decision making in nursing scale. In Measurement of Nursing Outcomes, C. Feher Waltz \& L. Sherman, Eds, Springer, New York, NY. 2001. PMid:11681561

[37] Upton DR, Upton PM. Development of an evidence-based practice questionnaire for nurses. Journal of Advanced Nursing. 2006; 54(4): 454-458. PMid:16448488 https://doi.org/10.1111/j. 1365-2648.2006.03739. $\mathrm{x}$

[38] Creswell JW, Plano Clark VL. Designing and conducting mixed methods research, 2nd ed. Thousand Oaks, CA; Sage; 2011.

[39] Morse J. Reframing rigour in qualitative inquiry. In N.K. Denzin \& Y.S. Lincoln (Eds.), The SAGE Handbook of Qualitative Research (5th ed) (pp.796-817). Thousand Oaks, CA: Sage; 2008.

[40] Loiselle CG, Profetto-McGrath J. Canadian essentials of nursing research, 2nd ed. New York: Lippincott Williams \& Wilkins. 2007.

[41] Gillespie M, Paterson B. Helping novice nurses make effective clinical decisions: The situated clinical decision-making framework. Nursing Education Perspectives. 2009; 30(3): 164-170. PMid:19606659

[42] Seright TJ. Clinical decision-making of rural novice nurses. Rural and Remote Health. 2011; 11: 1726. PMid:21787110

[43] Zsambok CE, Klein G. Naturalistic decision making. New York: Routledge; 2009.

[44] Rycroft-Malone J, Kitson A, Harvey G, et al. Ingredients for change: Revisiting a conceptual framework. Quality \& Safety in Health Care. 2002; 11(2): 174-180. https://doi.org/10.1136/qhc.11.2.1 74

Published by Sciedu Press 
[45] McCormack B, Kitson A, Harvey G, et al. Getting evidence into practice: The meaning of 'context'. Journal of Advanced Nursing. 2002; 38(1): 94-104. PMid:11895535 https://doi.org/10.104 $6 / j .1365-2648.2002 .02150 \cdot x$

[46] Hauck S, Winsett RP, Kuric J. Leadership facilitation strategies to establish evidence-based practice in an acute care hospital. Journal of Advanced Nursing. 2013; 69(3): 664-674. https://doi.org/ $10.1111 / j .1365-2648.2012 .06053 . x$

[47] Harvey G, Loftus-Hills A, Rycroft-Malone J, et al. Getting evidence into practice: The role and function of facilitation. Journal of Advanced Nursing. 2002; 37(6): 577-588. PMid:11879422 https://doi.org/10.1046/j.1365-2648.2002.02126.x

[48] Wales S, Kelly M, Wilson V, et al. Enhancing transformational facilitation skills for nurses seeking to support practice innovation. Contemporary Nurse. 2013; 44(2): 178-188. PMid:23869502 https://doi.org/10.5172/conu.2013.44.2.178
[49] Rather C, May D. Health care work environments, employee satisfaction, and patient safety: Care provider perspectives. Health Care Management Review. 2007; 32(1): 2-11. https ://doi .org/10.1 097/00004010-200701000-00002

[50] Van Bogaert P, Timmermans O, Mace Weeks S, et al. Nursing unit teams matter: Impact of unit-level nurse practice environment, nurse work characteristics, and burnout on nurse reported job outcomes, and quality of care, and patient adverse events-A cross-sectional survey. International Journal of Nursing Studies. 2014; 51: 1123-1134. PMid:24444772 https://doi.org/10.1016/j.ijnurstu. 201 3.12 .009

[51] Van Bogaert P, Kowalski C, Mace Weeks S, et al. The relationship between nurse practice environment, nurse work characteristics, burnout and job outcome and quality of nursing care: A crosssectional survey. International Journal of Nursing Studies. 2013; 50: 1667-1677. https://doi.org/10.1016/j.ijnurstu.2013.05 .010 